\title{
Market Impacts of Energy Storage in a Transmission-Constrained Power System
}

\author{
Vilma Virasjoki, Paula Rocha, Afzal S. Siddiqui, and Ahti Salo
}

\begin{abstract}
Environmental concerns have motivated governments in the European Union and elsewhere to set ambitious targets for generation from renewable energy (RE) technologies and to offer subsidies for their adoption along with priority grid access. However, because RE technologies like solar and wind power are intermittent, their penetration places greater strain on existing conventional power plants that need to ramp up more often. In turn, energy storage technologies, e.g., pumped hydro storage or compressed air storage, are proposed to offset the intermittency of RE technologies and to facilitate their integration into the grid. We assess the economic and environmental consequences of storage via a complementarity model of a stylized Western European power system with market power, representation of the transmission grid, and uncertainty in RE output. Although storage helps to reduce congestion and ramping costs, it may actually increase greenhouse gas emissions from conventional power plants in a perfectly competitive setting. Conversely, strategic use of storage by producers renders it less effective at curbing both congestion and ramping costs, while having no net overall impact on emissions.
\end{abstract}

Index Terms-Energy storage, wind power, complementarity modeling.

\section{NOMENCLATURE}

Indices and Sets

$a(s) \in \mathcal{S}$ : ancestor node of scenario tree node $s \in \mathcal{S}$

$e \in \mathcal{E}:=\{$ solar, wind $\}$ : renewable energy sources

$\mathcal{F}_{s} \in \mathcal{S}$ : descendant nodes of scenario tree node $s \in \mathcal{S}$

$i \in \mathcal{I}$ : power producers

$\ell \in \mathcal{L}:$ transmission lines

$n \in \mathcal{N}$ : power network nodes

$s \in \mathcal{S}$ : scenario tree nodes

$t \in \mathcal{T}$ : time periods

$t(s) \in \mathcal{T}$ : time period to which scenario tree node $s \in \mathcal{S}$ belongs

$u \in \mathcal{U}_{n, i}$ : conventional generation units of producer $i \in \mathcal{I}$ at network node $n \in \mathcal{N}$

V. Virasjoki is with the Systems Analysis Laboratory, Dept. of Mathematics and Systems Analysis, Aalto University School of Science, P.O. Box 11100, 00076 Aalto, Finland, e-mail: vilma.virasjoki@aalto.fi.

P. Rocha is with the Systems Analysis Laboratory, Dept. of Mathematics and Systems Analysis, Aalto University School of Science, P.O. Box 11100, 00076 Aalto, Finland and the Dept. of Statistical Science, University College London, U.K., email: p.rocha@ucl.ac.uk.

A.S. Siddiqui is with the Dept. of Statistical Science, University College London, U.K. and the Dept. of Computer and Systems Sciences, Stockholm University, Sweden, e-mail: afzal.siddiqui@ucl.ac.uk.

A. Salo is with the Systems Analysis Laboratory, Dept. of Mathematics and Systems Analysis, Aalto University School of Science, P.O. Box 11100, 00076 Aalto, Finland, e-mail: ahti.salo@aalto.fi.

The authors acknowledge the support of the STEEM Project funded through the Aalto Energy Efficiency Research Programme. Siddiqui is grateful for support from the RISKY-RES project (no. 228811) funded by the Research Council of Norway.

\section{Parameters}

$A_{s, n}^{e}$ : availability factor for renewable energy generation of type $e \in \mathcal{E}$ at node $n \in \mathcal{N}$ for scenario $s \in \mathcal{S}(-)$

$B_{n, n^{\prime}}$ : element $\left(n, n^{\prime}\right)$ of node susceptance matrix, where $n, n^{\prime} \in \mathcal{N}(1 / \Omega)$

$C_{n, i, u}^{\text {conv }}$ : generation cost of unit $u \in \mathcal{U}_{n, i}$ from producer $i \in \mathcal{I}$ at node $n \in \mathcal{N}(€ / \mathrm{MWh})$

$C_{n, i, u}^{\text {up }}$ : ramp-up cost of unit $u \in \mathcal{U}_{n, i}$ from producer $i \in \mathcal{I}$ at node $n \in \mathcal{N}(€ / \mathrm{MWh})$

$C^{\text {sto }}$ : cost of discharge from storage (€/MWh)

$D_{t, n}^{\text {int }}$ : intercept of linear inverse demand function at node $n \in \mathcal{N}$ in period $t \in \mathcal{T}(€ / \mathrm{MWh})$

$D_{t, n}^{\text {slp }}$ : slope of linear inverse demand function at node $n \in \mathcal{N}$ in period $t \in \mathcal{T}\left(€ / \mathrm{MWh}^{2}\right)$

$E^{\text {in }}:$ storage input efficiency (-)

$E^{\text {sto }}$ : hourly rate of storage decay (MW/MWh)

$\bar{G}_{n, i, u}^{\text {conv }}$ : maximum generation capacity of unit $u \in \mathcal{U}_{n, i}$ from producer $i \in \mathcal{I}$ at node $n \in \mathcal{N}$ (MW)

$\bar{G}_{n, i}^{e}:$ maximum generation capacity of producer $i \in \mathcal{I}$ for renewable energy type $e \in \mathcal{E}$ at node $n \in \mathcal{N}$ (MW)

$H_{\ell, n}$ : element $(\ell, n)$ of network transfer matrix, where $\ell \in \mathcal{L}$ and $n \in \mathcal{N}(1 / \Omega)$

$K_{\ell}$ : maximum capacity of power line $\ell \in \mathcal{L}(\mathrm{MW})$

$P_{s}:$ probability of scenario $s \in \mathcal{S}(-)$

$R^{\text {in }}$ : hourly rate at which storage can be charged (MW/MWh) $R^{\text {out}}$ : hourly rate at which storage can be discharged (MW/MWh)

$S_{n} \in\{0,1\}$ : dummy parameter for slack node, where $n \in \mathcal{N}$ $(-)$

$\bar{R}_{n, i}$ : maximum storage capacity of producer $i \in \mathcal{I}$ at node $n \in \mathcal{N}$ (MWh)

$\underline{R}_{n, i}$ : minimum storage capacity of producer $i \in \mathcal{I}$ at node $n \in \mathcal{N}(\mathrm{MWh})$

$T_{t}$ : duration of period $t \in \mathcal{T}(\mathrm{h})$

\section{Primal Variables}

$g_{s, n, i, u}^{\text {conv }}$ : generation at node $n \in \mathcal{N}$ by producer $i \in \mathcal{I}$ using unit $u \in \mathcal{U}_{n, i}$ for scenario $s \in \mathcal{S}$ (MWh)

$g_{s, n, i}^{e}$ : renewable energy generation of type $e \in \mathcal{E}$ at node $n \in \mathcal{N}$ by producer $i \in \mathcal{I}$ for scenario $s \in \mathcal{S}(\mathrm{MWh})$

$g_{s, n, i, u}^{\text {up }}$ : generation ramp-up at node $n \in \mathcal{N}$ by producer $i \in \mathcal{I}$ using unit $u \in \mathcal{U}_{n, i}$ for scenario $s \in \mathcal{S}$ (MWh)

$q_{s, n, i}$ : sales at node $n \in \mathcal{N}$ by producer $i \in \mathcal{I}$ for scenario $s \in \mathcal{S}(\mathrm{MWh})$

$r_{s, n, i}$ : (end-of-period) stored energy by producer $i \in \mathcal{I}$ at node $n \in \mathcal{N}$ for scenario $s \in \mathcal{S}(\mathrm{MWh})$

$r_{s, n, i}^{\text {in }}$ : energy charged into storage at node $n \in \mathcal{N}$ by producer $i \in \mathcal{I}$ for scenario $s \in \mathcal{S}(\mathrm{MWh})$ 
$r_{s, n, i}^{\text {out }}$ : energy discharged from storage at node $n \in \mathcal{N}$ by producer $i \in \mathcal{I}$ for scenario $s \in \mathcal{S}$ (MWh)

$v_{s, n}$ : voltage angle at node $n \in \mathcal{N}$ for scenario $s \in \mathcal{S}$ (rad)

\section{Dual Variables}

$\beta_{s, n, i, u}^{\text {conv }}$ : shadow price on generation capacity at node $n \in \mathcal{N}$ for conventional generation unit $u \in \mathcal{U}_{n, i}$ of producer $i \in \mathcal{I}$ and scenario $s \in \mathcal{S}(€ / \mathrm{MWh})$

$\beta_{s, n, i}^{e}$ : shadow price on renewable energy generation capacity at node $n \in \mathcal{N}$ for energy source type $e \in \mathcal{E}$, producer $i \in \mathcal{I}$ and scenario $s \in \mathcal{S}(€ / \mathrm{MWh})$

$\beta_{s, n, i, u}^{\text {up }}$ : shadow price on ramp-up constraint at node $n \in \mathcal{N}$ for conventional generation unit $u \in \mathcal{U}_{n, i}$ of producer $i \in \mathcal{I}$ and scenario $s \in \mathcal{S}(€ / \mathrm{MWh})$

$\gamma_{s, n}$ : dual for slack node $n \in \mathcal{N}$ and scenario $s \in \mathcal{S}(-)$

$\theta_{s, i}$ : shadow price on energy balance for producer $i \in \mathcal{I}$ and scenario $s \in \mathcal{S}(€ / \mathrm{MWh})$

$\lambda_{s, n, i}^{\mathrm{bal}}$ : shadow price on stored energy balance at node $n \in \mathcal{N}$ for producer $i \in \mathcal{I}$ and scenario $s \in \mathcal{S}$ (€/MWh)

$\lambda_{s, n, i}^{\text {in }}$ : shadow price on maximum storage charging at node $n \in \mathcal{N}$ for producer $i \in \mathcal{I}$ and scenario $s \in \mathcal{S}$ (€/MWh)

$\lambda_{s, n, i}^{\mathrm{lb}}, \lambda_{s, n, i}^{\mathrm{ub}}$ : shadow price on energy storage capacity at node $n \in \mathcal{N}$ for producer $i \in \mathcal{I}$ and scenario $s \in \mathcal{S}$ (€/MWh)

$\lambda_{s, n, i}^{\text {out }}$ : shadow price on maximum storage discharging at node $n \in \mathcal{N}$ for producer $i \in \mathcal{I}$ and scenario $s \in \mathcal{S}$ (€/MWh)

$\bar{\mu}_{s, \ell}, \underline{\mu}_{s, \ell}:$ shadow price on transmission capacity for transmission line $\ell$ and scenario $s \in \mathcal{S}(€ / \mathrm{MW})$

$\omega_{s, n}$ : congestion charge at node $n \in \mathcal{N}$ for scenario $s \in \mathcal{S}$ $(€ / \mathrm{MWh})$

\section{INTRODUCTION}

\section{A. Background}

In order to mitigate climate change, governments have imposed increasingly stringent restrictions on $\mathrm{CO}_{2}$ emissions and introduced policies to incentivize the adoption of renewable energy (RE) technologies. For example, in the European Union (EU), one of the 20-20-20 climate change targets requires all member states to reduce their $\mathrm{CO}_{2}$ emissions by $20 \%$ by the year 2020 relative to 1990 levels [1] with more ambitious targets proposed from 2020 onward [2]. However, given that electricity industries in most OECD countries have been deregulated with the aim of improving economic efficiency [3], investment and operational decisions are typically undertaken by profit-maximizing companies that may balk at adopting socially desirable but costly RE technologies. Thus, in order to entice power companies to invest in RE technologies, policymakers have both introduced new regulation, e.g., priority grid access, and provided economic incentives, e.g., feed-in tariffs and renewable energy certificates [4].

While such measures have increased RE technology output, e.g., to over $25 \%$ of German electricity generation in 2013, and reduced $\mathrm{CO}_{2}$ emissions, e.g., by nearly $30 \%$ in 2013 relative to 1990 levels, further penetration of such resources may be limited by the complexities of integrating them into the grid. Moreover, because RE technologies like solar and wind power are intermittent, their adoption implies that existing conventional power plants must ramp more frequently. In order to address this difficulty, energy storage technologies, e.g., pumped hydro storage (PHS) or compressed air storage, have been proposed to offset the intermittency of RE technologies. Together with subsequent expansion and reinforcement of the transmission grid itself [5], [6], better techniques for congestion management [7], and enhanced demand response [8], storage is likely to facilitate an economically viable way to integrate intermittent RE technologies. Yet, a deeper understanding of the economic and environmental impacts of storage coupled with RE technologies is required for crafting policy.

\section{B. Literature Review}

Previous work, such as [9], has shown that storage in a power system can have welfare-enhancing effects, albeit at the expense of producers as it reduces the price differential between peak and off-peak periods. Moreover, [10] has demonstrated that when storage belongs to producers behaving à la Cournot, they are able to use it to move energy from peak to off-peak periods, thereby increasing peak prices above competitive levels. Sioshansi [11] has concluded, using a stylized model without transmission constraints or uncertainty, that strategic generators with standalone storage or generatorowned storage may cause social welfare to decrease. Focusing on emissions, a supply-function equilibrium model of ERCOT illustrates that greenhouse gas (GHG) emissions may increase in the presence of both wind power and storage [12].

In contrast to these papers, which do not represent the transmission network, [13] examine electricity storage with transmission constraints. By formulating a mixed-complementarity problem (MCP) [14], they determine how storage affects the formation of locational marginal prices. Consequently, the optimal locations and sizes of storage systems can be obtained from such a modeling approach. Likewise, [15] use a DC optimal power flow (OPF) model to assess storage technology portfolios in a transmission-constrained network. In an analysis of battery storage, [16] perform a case study of the PJM market using a unit-commitment model to show the potential for modest savings for consumers, which are more than offset by capital expenditures for energy storage and lower profits for generators along with increased $\mathrm{CO}_{2}$ emissions. Similar results for a 25-node test network are reported by [17] using an OPF model. However, [13], [15], [16], and [17] consider neither uncertainty in RE output nor market power. Therefore, we aim to investigate the economic and environmental impacts of energy storage using a realistic test network that incorporates features such as market power and uncertainty in RE output.

\section{Research Objectives and Contribution}

Using a complementarity approach [14], we model the decision-making problems of power producers and the grid owner. The former decide how to operate power plants (both conventional and RE) and storage in order to maximize expected profit inclusive of congestion payments to transmit electricity between nodes. Uncertainty in RE output is represented via discrete scenarios that evolve over several hours of a typical day, while inventory-balance constraints regulate charging and discharging decisions for storage. Meanwhile, 
the grid owner decides how much electricity to import at each node in order to maximize revenues from congestion fees [18].

We implement the resulting MCP for a 15-node (and 28line) test network of Western Europe [19] over four hours corresponding to the critical morning ramping periods for conventional power plants with intermittent RE output represented by discrete scenarios in each hour. We estimate installed capacities in Belgium, France, Germany, and the Netherlands as well as representations of demand and transmission interconnections. A linearized direct current (DC) load-flow approximates physical flows of electricity in the network.

We demonstrate that while storage facilitates the integration of RE technologies by reducing network congestion and ramping costs, it may actually result in higher $\mathrm{CO}_{2}$ emissions. This somewhat counterintuitive result occurs because conventional power plants are used during early-morning hours to charge storage for use later in the day when RE output may not be adequate. In turn, peak-hour conventional generation is reduced, but the corresponding reduction in $\mathrm{CO}_{2}$ emissions does not offset its increase during off-peak hours due to the efficiency losses associated with storage and the displacement of gas-fired generation (used for ramping without storage) by coal plants (used for charging storage).

By contrast, storage does not significantly affect $\mathrm{CO}_{2}$ emissions when producers exercise market power. In effect, producers have reduced incentives for using storage as it depresses peak-hour prices. This suggests that the environmental effects of storage may not be detrimental in practice because of the presence of market power. Nevertheless, the results are for a stylized example during a specific period of the day, and policymakers should consider such unintended environmental consequences when setting targets for RE generation. Indeed, [20] demonstrates with a two-node example that $\mathrm{CO}_{2}$ emissions may increase once a $\mathrm{CO}_{2}$ tax is introduced as it makes the coal power plant at the exporting node relatively more expensive than the gas-fired plant at the importing node. Hence, the resulting alleviation of congestion on the transmission line effectively replaces two monopolies with a single duopoly, which leads to lower prices and increased consumption to the extent that $\mathrm{CO}_{2}$ emissions rise overall.

\section{Structure of the Paper}

In Section II, we present our modeling assumptions and the mathematical formulation for the MCP that captures production, sales, storage, and transmission decisions. Next, in Section III, we implement numerical examples on a Western European test network to gain insights about how market power, storage, and wind output interact. We conclude the paper in Section IV by summarizing our main points, discussing the limitations of our approach, and outlining directions for future research.

\section{PROBlem Formulation}

\section{A. Assumptions}

Following the standard approach in power system economics [18], we use a DC load-flow approximation in representing transmission flows. Here, the susceptance, $B_{n, n^{\prime}}$, and network transfer, $H_{\ell, n}$, parameters capture power transfer distribution factors. Given voltage angles, $v_{s, n}$, and the declaration of a slack node (with zero voltage angle), the power flow on line $\ell$ is $\sum_{n \in \mathcal{N}} H_{\ell, n} v_{s, n}$ and the imported energy at node $n$ is $\sum_{n^{\prime} \in \mathcal{N}} T_{t(s)} B_{n^{\prime}, n} v_{s, n^{\prime}}$ in scenario $s$.

Each producer may own both conventional and RE plants as well as energy storage facilities anywhere in the network. As we will describe in Section III, storage capacities are assigned to generators based on current market conditions in Western Europe. However, following [11], storage facilities could be standalone or managed by a welfare-maximizing independent system operator (ISO). In the case of perfect competition, this would yield the same results as in our setup but would mitigate the exercise of market power by strategic producers in the case of Cournot oligopoly. The conventional units with installed capacities, $\bar{G}_{n, i, u}^{\text {conv }}$, have generation costs of $C_{n, i, u}^{\text {conv }}$ and ramp-up costs of $C_{n, i, u}^{\text {up }}$ to reflect the wear and tear on turbines from changes in operating levels. RE plants with installed capacities, $\bar{G}_{n, i}^{\mathrm{e}}$, have no operating costs but are non-dispatchable, i.e., their output depends solely on stochastic availability factors, $A_{s, n}^{\mathrm{e}}$, for each scenario $s$ and node $n$ with probability $P_{s}$. Producers may inject or withdraw energy from storage at withdrawal cost $C^{\text {sto }}$ as long as they respect the maximum charging $\left(R^{\text {in }}\right)$ and discharging $\left(R^{\text {out }}\right)$ rates along with the minimum $\left(\underline{R}_{n, i}\right)$ and maximum $\left(\bar{R}_{n, i}\right)$ storage capacities. There are also losses associated with both the charging $\left(E^{\text {in }}\right)$ and storage $\left(E^{\text {sto }}\right)$ processes. Finally, each producer may generate electricity at one node and sell it (or store it) at another one provided that it pays to access transmission capacity.

By selling electricity at node $n$ in scenario $s$, producer $i$ receives a price that depends on the linear inverse demand curve, i.e., $D_{t(s), n}^{\mathrm{int}}-D_{t(s), n}^{\mathrm{slp}} \sum_{i^{\prime} \in \mathcal{I}} q_{s, n, i^{\prime}}$, where $\sum_{i^{\prime} \in \mathcal{I}} q_{s, n, i^{\prime}}$ is the total sales (and consumption). Under perfect competition, each producer treats the price at each node as a constant.

As in [18], we assume that the grid owner controls transmission flows to maximize its expected revenues from congestion charges paid on imports at each node while respecting the thermal limits of the transmission lines. The congestion charge, $\omega_{s, n}$, is the shadow price on the market-clearing condition that balances supply and demand at each node. Hence, a producer that generates electricity at node $n$ and sells it at node $n^{\prime}$ receives $\omega_{s, n}$ to send electricity from node $n$ to a hub node and pays $\omega_{s, n^{\prime}}$ to send electricity from the hub to node $n^{\prime}$ for a net congestion payment of $\omega_{s, n^{\prime}}-\omega_{s, n}$.

\section{B. Decision-Making Problems and Equilibrium Conditions}

1) Producers' Problems: Each producer $i \in \mathcal{I}$ finds generation (both conventional and RE), storage, and sales levels to maximize its expected profit. The profit consists of sales revenues less generation and ramp-up costs incurred by conventional generation units, storage costs, and net congestion payments for electricity transmission between nodes (1). Each producer's profits are represented in terms of the point-to-point congestion charges, which enables the effect of market power on sales to be captured through each producer's revenue at 
each node inclusive of congestion charges.

$$
\begin{aligned}
& \max _{x} \sum_{s \in \mathcal{S}} \sum_{n \in \mathcal{N}} P_{s}\left[\left(D_{t(s), n}^{\mathrm{int}}-D_{t(s), n}^{\mathrm{slp}} \sum_{i^{\prime} \in \mathcal{I}} q_{s, n, i^{\prime}}-\omega_{s, n}\right) q_{s, n, i}\right. \\
& -\sum_{u \in \mathcal{U}_{n, i}}\left(C_{n, i, u}^{\mathrm{conv}}-\omega_{s, n}\right) g_{s, n, i, u}^{\mathrm{conv}} \\
& -\sum_{u \in \mathcal{U}_{n, i}} C_{n, i, u}^{\mathrm{up}} g_{s, n, i, u}^{\mathrm{up}}+\omega_{s, n} \sum_{e \in \mathcal{E}} g_{s, n, i}^{e} \\
& \left.-\omega_{s, n} r_{s, n, i}^{\text {in }}-\left(C^{\text {sto }}-\omega_{s, n}\right) r_{s, n, i}^{\text {out }}\right] \\
& \text { s.t. } \sum_{n \in \mathcal{N}} q_{s, n, i}=\sum_{n \in \mathcal{N}} \sum_{u \in \mathcal{U}_{n, i}} g_{s, n, i, u}^{\text {conv }}+\sum_{n \in \mathcal{N}} \sum_{e \in \mathcal{E}} g_{s, n, i}^{e} \\
& +\sum_{n \in \mathcal{N}} r_{s, n, i}^{\text {out }}-\sum_{n \in \mathcal{N}} r_{s, n, i}^{\text {in }}\left(\theta_{s, i}\right), \forall s \\
& g_{s, n, i, u}^{\mathrm{conv}} \leq T_{t(s)} \bar{G}_{n, i, u}^{\mathrm{conv}}\left(\beta_{s, n, i, u}^{\mathrm{conv}}\right), \forall n, s, \forall u \in \mathcal{U}_{n, i} \\
& g_{s, n, i, u}^{\text {conv }}-g_{a(s), n, i, u}^{\text {conv }} \leq g_{s, n, i, u}^{\text {up }}\left(\beta_{s, n, i, u}^{\text {up }}\right) \text {, } \\
& \forall n, s, \forall u \in \mathcal{U}_{n, i} \\
& g_{s, n, i}^{e}=T_{t(s)} A_{s, n}^{e} \bar{G}_{n, i}^{e}\left(\beta_{s, n, i}^{e}\right), \forall e, n, s \\
& r_{s, n, i}=T_{t(s)}\left(1-E^{\text {sto }}\right) r_{a(s), n, i}+E^{\text {in }} r_{s, n, i}^{\text {in }} \\
& -r_{s, n, i}^{\mathrm{out}}\left(\lambda_{s, n, i}^{\mathrm{bal}}\right), \forall n, s \\
& r_{s, n, i}^{\text {in }} \leq T_{t(s)} R^{\text {in }} \bar{R}_{n, i}\left(\lambda_{s, n, i}^{\text {in }}\right), \forall n, s \\
& r_{s, n, i}^{\text {out }} \leq T_{t(s)} R^{\text {out }} \bar{R}_{n, i}\left(\lambda_{s, n, i}^{\text {out }}\right), \forall n, s \\
& \underline{R}_{n, i} \leq r_{s, n, i} \leq \bar{R}_{n, i}\left(\lambda_{s, n, i}^{\mathrm{lb}}, \lambda_{s, n, i}^{\mathrm{ub}}\right), \forall n, s \\
& g_{s, n, i, u}^{\text {conv }}, g_{s, n, i, u}^{\text {up }} \geq 0, \forall n, s, \forall u \in \mathcal{U}_{n, i} ; g_{s, n, i}^{e} \geq 0, \forall e, n, s \text {; } \\
& q_{s, n, i}, r_{s, n, i}, r_{s, n, i}^{\text {in }}, r_{s, n, i}^{\text {out }} \geq 0, \forall n, s
\end{aligned}
$$

Here, $x:=\left\{g_{s, n, i, u}^{\text {conv }}, g_{s, n, i}^{e}, g_{s, n, i, u}^{\text {up }}, q_{s, n, i}, r_{s, n, i}, r_{s, n, i}^{\text {in }}, r_{s, n, i}^{\text {out }}\right.$ : $\left.e \in \mathcal{E}, i \in \mathcal{I}, n \in \mathcal{N}, s \in \mathcal{S}, u \in \mathcal{U}_{n, i}\right\}$, and dual variables are in brackets next to their corresponding constraints. Constraints (2) ensure energy balance between total sales and total (conventional and RE) generation plus total net discharge from storage in each scenario. For each conventional generation unit, capacity generation (3) and ramp-up (4) constraints are imposed, while RE generation depends simply on resource availability (5). While RE outputs are decision variables, they are effectively exogenous because output equals to availability in each scenario. Thus, although substantial RE capacity may belong to fringe producers, they are unable to use it to withhold sales. Furthermore, any strategic producers with RE capacity cannot use it to exert market power. Restrictions (6) guarantee that the electric energy available in storage at the end of a given period is equal to the energy stored at the end of the previous period plus the energy charged into storage minus the energy discharged from storage during that period. Constraints (7) and (8) impose an upper limit on the amount of electricity that can be charged into and discharged from storage, respectively, whereas constraints (9) guarantee that the amount of electricity stored remains within certain limits.

2) Grid Owner's Problem: The grid owner determines how much power to import at each network node in order to maximize its expected net payments from congestion charges:

$$
\begin{aligned}
\max _{v_{s, n}} & \sum_{s \in \mathcal{S}} \sum_{n \in \mathcal{N}} P_{s} \omega_{s, n} \sum_{n^{\prime} \in \mathcal{N}} T_{t(s)} B_{n, n^{\prime}} v_{s, n^{\prime}} \\
\text { s.t. } & \sum_{n \in \mathcal{N}} H_{\ell, n} v_{s, n} \leq K_{\ell}\left(\bar{\mu}_{s, \ell}\right), \forall \ell, s \\
& -\sum_{n \in \mathcal{N}} H_{\ell, n} v_{s, n} \leq K_{\ell}\left(\underline{\mu}_{s, \ell}\right), \forall \ell, s \\
& S_{n} v_{s, n}=0\left(\gamma_{s, n}\right), \forall n, s \\
& v_{s, n} \text { u.r.s., } \forall n, s,
\end{aligned}
$$

where u.r.s. stands for "unrestricted in sign." Reflecting the DC load-flow approximation, constraints (12)-(13) guarantee that the power flow on each transmission line remains within the line's capacity limit, whereas (14) defines the slack node of the network.

3) Market-Clearing Conditions: The market-clearing conditions ensure that, in each network node and each scenario, the energy demand (equal to sales) is met by energy produced plus net energy discharged from storage at that node and net energy imports from other network nodes.

$$
\begin{gathered}
\sum_{i \in \mathcal{I}} q_{s, n, i}-\sum_{i \in \mathcal{I}} \sum_{u \in \mathcal{U}_{n, i}} g_{s, n, i, u}^{\text {conv }}-\sum_{i \in \mathcal{I}} \sum_{e \in \mathcal{E}} g_{s, n, i}^{e}-\sum_{i \in \mathcal{I}} r_{s, n, i}^{\text {out }} \\
+\sum_{i \in \mathcal{I}} r_{s, n, i}^{\text {in }}-\sum_{n^{\prime} \in \mathcal{N}} T_{t(s)} B_{n, n^{\prime}} v_{s, n^{\prime}}=0 \text {, with } \omega_{s, n} \text { u.r.s., } \forall n, s
\end{gathered}
$$

\section{Mixed Complementarity Problem}

In order to find a market-equilibrium solution, we formulate a stochastic MCP by taking the Karush-Kuhn-Tucker (KKT) conditions for the producers' and grid owner's optimization problems defined in (1)-(10) and (11)-(15), respectively, along with the market-clearing constraints (16). The aforementioned KKT conditions are presented in the Appendix. We remark that, like in [18], our MCP can be analogously formulated as a convex quadratic program (QP), and, thus, the globally optimal solution to the equivalent QP [14, Theorem 4.4] also provides an equilibrium to the MCP.

\section{NUMERICAL EXAMPLES}

\section{A. Data}

We implement our model for a stylized 15-node network representing Western Europe (Fig. 1). The network parameters, such as line capacities and the node structure, are based on [19]. Nodes $n 1-n 7$ represent actual generation and load, while $n 8-n 15$ are auxiliary nodes without any supply or demand that are required for modeling cross-border flows.

Uncertainty in wind and solar generation is modeled by a scenario tree with equiprobable scenario paths (Fig. 2). The time frame represents the four-hour morning ramp during which producers make critical generation dispatch and storage decisions. The solar and wind availabilities represent the situation in June/July 2011 based on German data from the EEX. Using the average hourly solar generation in Fig. 3, we calculate an hourly solar availability factor by dividing the mean observed output by the German installed capacity 
(25 GW, see Table II). This yields mean availability factors of $0.002,0.019,0.064$, and 0.135 for hours $t 5-t 8$ along with standard deviations of $0.001,0.007,0.021$, and 0.043 . Our scenarios in Fig. 2 approximate these summary statistics: the mean availability factors are $0,0.025,0.075$, and 0.150 with standard deviations of $0,0.021,0.025$, and 0.047 . The expected wind availability factor for each hour is obtained from the average observed wind production in Fig. 4. Since installed German wind capacity is 29 GW (see Table II), the mean availability factor during June/July 2011 for each of the four hours, $t 5-t 8$, corresponds to approximately $14 \%$ of the total installed capacity. The corresponding standard deviation of the wind availability factor in each hour is about $10 \%$. Using this information, the scenarios are constructed such that (a) the expected wind generation is the same in each hour (approximately 14\% of installed capacity) while (b) the correlation between solar and wind remains low. For example, during hour $t 7$, scenario $s 4$ is based on $6 \mathrm{GWh}$ of output, which yields an availability factor of 0.21 . By contrast, scenario $s 7$ has $2 \mathrm{GWh}$ of output (resulting in an availability factor of 0.07), whereas both scenarios $s 5$ and $s 6$ have 4 $\mathrm{GWh}$ of output (resulting in an availability factor of 0.14 ). The standard deviation of possible wind availability factors in Fig. 2 varies between 5\% and 7\% during each hour (excluding t5), which is roughly in line with the observed data. The assumed increase in demand in relation to average hourly demand, a stylized load profile from $t 5$ to $t 8$, is: $0.84,0.92,1.01,1.07$.

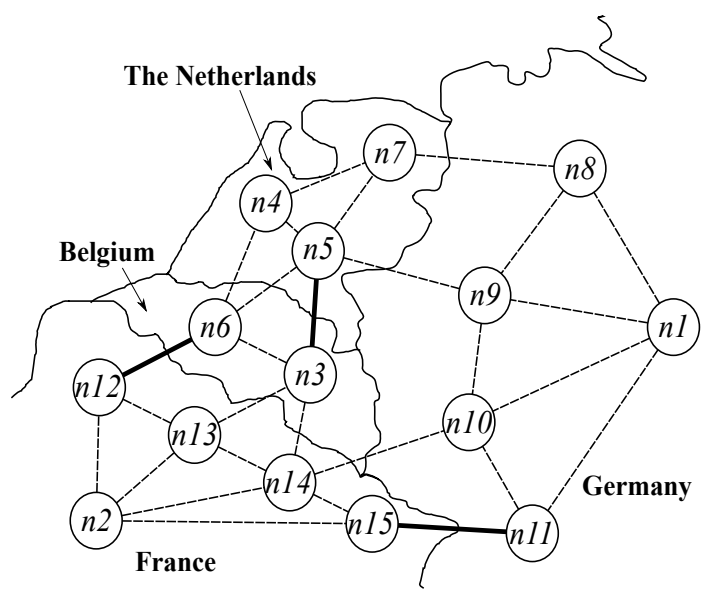

Fig. 1. Stylized Western European test network [19]

Market characteristics, nodal reference demands, and reference prices are calibrated to estimated linear inverse demand functions. The annual average hourly loads (in GW) are 62, $55,2,8,3,8$, and 3 for nodes $n 1-n 7$, respectively, which are based on [23] for 2011. Correspondingly, the weighted average price is $€ 50.2 / \mathrm{MWh}$. Price elasticity at the reference point is assumed to be -0.25 [23], and details about the parameter calculation of the linear inverse demand function are in [24].

On the supply side, generation costs for technologies in Table I are based on [19] and the merit-order curve of [23]. The complexity of generation processes causes additional technology-specific ramping costs when output is adjusted. These costs are driven by the decreased fuel efficiency com-

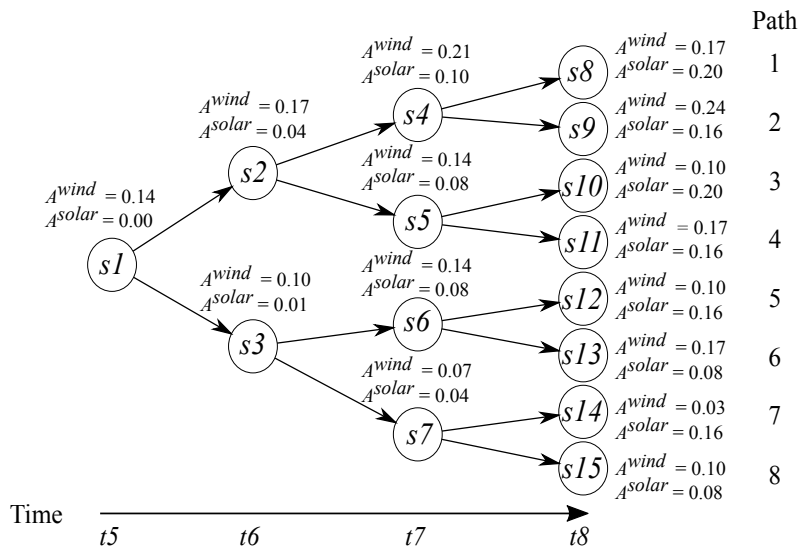

Fig. 2. Scenario tree for wind and solar generation availability as a fraction of installed capacity

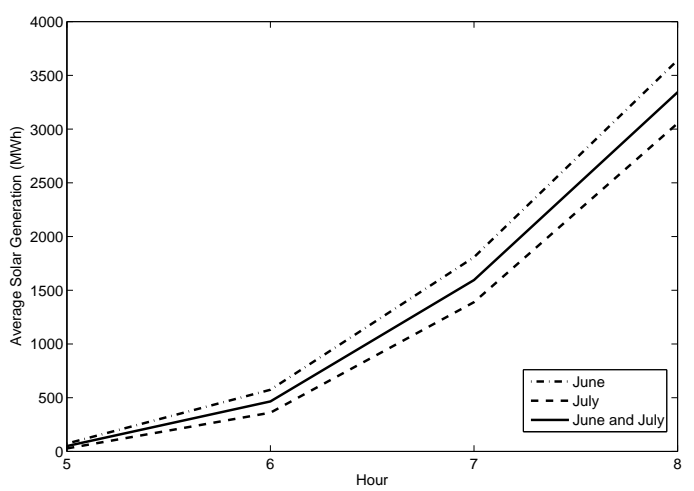

Fig. 3. Average hourly solar generation in Germany for 2011 [21]

pared to constant generation, increased stress on generators' components, and replacement costs. We use stylized ramp-up costs [25] that prevent unrealistic ramping. Thus, the observed ramping rates (as a percentage of total generation) in our model are in line with most plants' ramping feasibilities [26], [27]. One distinction is that neither [26] nor [27] allows for nuclear plants to ramp. However, in France, load following by nuclear plants is required because they generate over $75 \%$ of

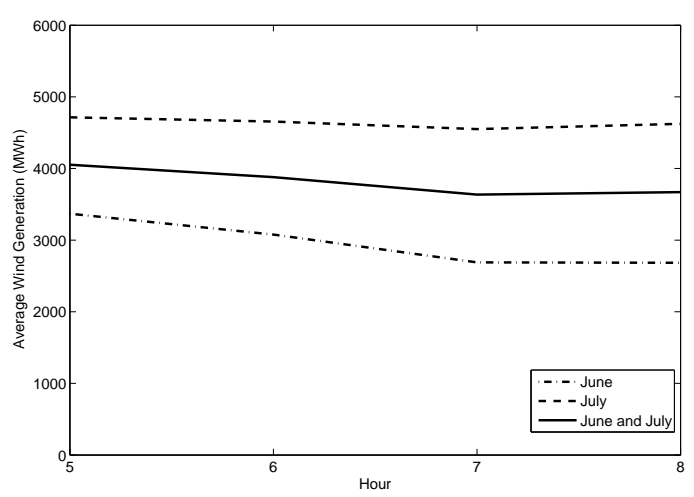

Fig. 4. Average hourly wind generation in Germany for 2011 [22] 
TABLE I

MARGINAL COST, RAMP-UP COST, AND $\mathrm{CO}_{2}$ EMISSIONS RATE

\begin{tabular}{|l|c|c|c|}
\hline Type & $\begin{array}{c}\text { Marginal cost } \\
(€ / \mathrm{MWh})\end{array}$ & $\begin{array}{c}\text { Ramp-up cost } \\
(€ / \mathrm{MWh})\end{array}$ & $\begin{array}{c}\mathrm{CO}_{2} \text { emissions per } \\
\text { unit of electricity } \\
\text { output }(\mathrm{kg} / \mathrm{kWh})\end{array}$ \\
\hline$u 1$ (nuclear) & 10 & 6.7 & 0 \\
$u 2$ (lignite) & 20 & 6.7 & 0.94 \\
$u 3$ (coal) & 22 & 4.7 & 0.83 \\
$u 4$ (CCGT) & 30 & 5.8 & 0.37 \\
$u 5$ (gas) & 45 & 2.3 & 0.50 \\
$u 6$ (oil) & 60 & 2.3 & 0.72 \\
$u 7$ (hydro) & 0 & 6.7 & 0 \\
\hline
\end{tabular}

TABLE II

ESTIMATED INSTALLED GENERATION CAPACITY OF PRODUCERS (GW) IN 2011 AND AVAILABILITY PERCENTAGES PER TECHNOLOGY

\begin{tabular}{|cl|ccccccccc|}
\hline \multicolumn{2}{|c|}{ Node Producer } & $u 1$ & $u 2$ & $u 3$ & $u 4$ & $u 5$ & $u 6$ & $u 7$ & $\mathrm{~S}$ & $\mathrm{~W}$ \\
\hline$n 1$ & E.ON & 5 & 1 & 5 & 2 & 2 & 1 & - & - & - \\
& RWE & 4 & 10 & 5 & 3 & 3 & - & 0.5 & - & 0.5 \\
& EnBW & 3 & - & 4 & 0.5 & - & 0.5 & 0.5 & - & - \\
& Vattenfall & - & 9 & 2 & 1 & 1 & 0.5 & - & - & - \\
& FringeD & - & - & 9 & 4.5 & 4 & 2 & 1 & 25 & 28.5 \\
\hline$n 2$ & EDF & 63 & - & 4 & - & - & 7 & 15 & - & 0.5 \\
& FringeF & - & 2 & - & 3 & 2 & - & 2 & - & 6.5 \\
\hline$n 3$, & Electrabel & 4 & - & - & 3 & 1 & - & - & - & 0.5 \\
$n 6$ & FringeB & 2 & - & 1 & 1 & - & - & - & 2 & 0.5 \\
\hline$n 4$, & Electrabel & - & - & 1 & 3 & 2 & - & - & - & 0.5 \\
$n 5$, & Essent & - & - & 1 & 1.5 & - & - & - & - & - \\
$n 7$ & Nuon & - & - & 1 & 2 & 1 & - & - & - & 0.5 \\
& FringeN & - & - & 1 & 3.5 & 2 & - & - & - & 1.5 \\
\hline \hline \multirow{2}{*}{$\%$} & Available & 80 & 85 & 84 & 89 & 86 & 86 & 30 & Fig. & Fig. \\
& & & & & & & \\
&
\end{tabular}

the country's electricity. Meanwhile, load following by nuclear plants in Germany is increasingly common because of the need to avoid negative electricity prices [28]. Related work on the use of storage in Europe likewise allows for nuclear plants to ramp up and down by $5 \%$ and $10 \%$ of rated capacity, respectively [9]. The $\mathrm{CO}_{2}$ emissions of fossil fuels in Table I are estimated from average plant efficiencies and emissions factors reported in [23] and [29], respectively.

Table II estimates producers' installed generation capacities. Companies with the largest national shares of power production are taken into account with the remaining capacity allocated to a fringe. Figures are estimates for 2011 gathered from companies' websites and annual activity reports so that the aggregated capacities match national capacity in [23]. The stylized data do not permit in-depth firm-level analyses, but they help to assess aggregate market outcomes. To account for plants' offline time, such as outages and revisions, only a defined share of the installed capacity for each technology is assumed to be available for generation.

Estimates for installed storage capacities in the year 2014 are based on operational installations' power in 2014 [30]. At node $n 1$, E.ON, RWE, EnBW, Vattenfall, and a fringe of German producers own $5,11,1,16$, and $3 \mathrm{GWh}$, respectively.
EDF owns $30 \mathrm{GWh}$ at node $n 2$, and Electrabel owns a combined $6 \mathrm{GWh}$ at nodes $n 3$ and $n 6$. There is insignificant grid-connected storage in the Netherlands, and over $90 \%$ of the storage capacity in the other three countries is PHS.

The cost of storage discharge, $C^{\text {sto }}$, is assumed to be zero because most of the current capacity is PHS [9]. Other storage parameter assumptions are $E^{\text {sto }}=0, E^{\text {in }}=0.75$, $R^{\text {in }}=0.16$, and $R^{\text {out }}=0.16$. We assume no losses for stored electricity on an hourly basis but account for round-trip efficiencies via $E^{\text {in }}<1$. Furthermore, for each node $n$ and producer $i$, the minimum storage capacity, $\underline{R}_{n, i}$, is calculated as $\underline{R}_{n, i}=0.3 \bar{R}_{n, i}$, which is also the initial storage level.

\section{B. Computational Issues}

We present numerical results for four cases: perfect competition (PC) with and without storage capacity in the grid and likewise for Cournot oligopoly (CO). The model and corresponding cases are implemented in GAMS, and the resulting MCP is solved with CPLEX after re-formulation as a QP problem in less than one minute on a workstation using a $3.40 \mathrm{GHz}$ Intel i7-4770 core processor and $8 \mathrm{~GB}$ of RAM.

\section{Results}

The price-smoothing effect of storage from [9] is corroborated in Fig. 5. The impact is roughly similar in off-peak periods $(t 5-t 6)$ for both PC and $\mathrm{CO}$ but is much larger under PC during peak hours ( $t 7-t 8)$. This is because Cournot producers have the incentive to withhold sales, which diminishes the price decrease and yields higher profits in peak hours.

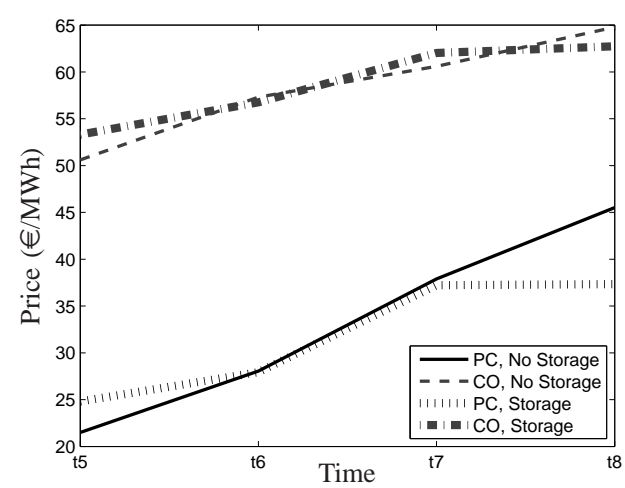

Fig. 5. Expected prices over all nodes and scenarios

Fig. 6 illustrates total expected a) conventional generation $\left(g^{\text {conv }}\right)$, b) ramping $\left(g^{\text {up }}\right)$ and c) $\mathrm{CO}_{2}$ emissions by technology type in off-peak $(t 5-t 6)$ and peak $(t 7-t 8)$ periods. In addition, d) total expected stored energy $(r)$ is presented. We observe that Cournot producers withhold their output and use less storage in comparison to the perfectly competitive situation. The results also demonstrate that producers with storage increase their conventional production in off-peak hours to store energy and, consequently, reduce conventional production during peak hours due to storage discharge.

Fig. $6 \mathrm{~b}$ shows that producers with storage rely less on ramping their conventional production to meet the higher 

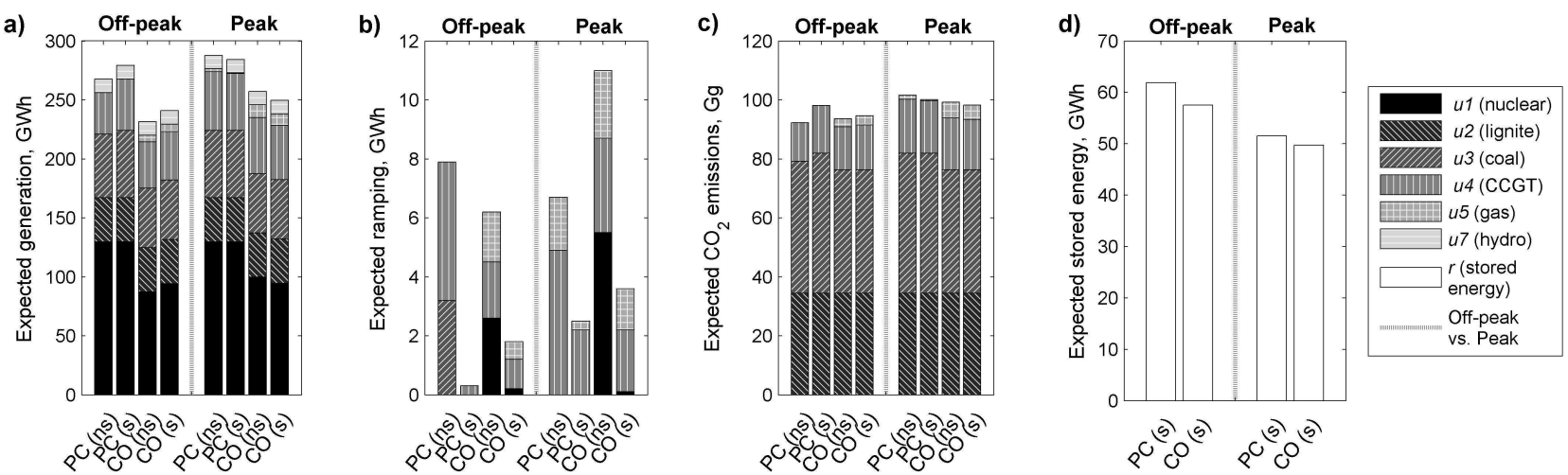

Fig. 6. Expected a) conventional generation (GWh), b) ramping (GWh), c) $\mathrm{CO}_{2}$ emissions $(\mathrm{Gg})$, and d) stored energy (GWh) in off-peak ( $\left.t 5-t 6\right)$ and peak hours $(t 7-t 8)$ for PC and $\mathrm{CO}$ with (s) and without (ns) storage cases

demand of peak periods. As presented in Fig. 7, this gives significant savings in ramping costs of $74 \%$ and $80 \%$ for CO and $\mathrm{PC}$, respectively. Indeed, the possibility to use storage removes an obstacle to integrating intermittent RE by lowering ramping costs for producers. More ramping is used under $\mathrm{CO}$ than under PC when producers have storage. Consequently, under $\mathrm{CO}$, ramping accounts for a higher relative share of total supply. This can result from a situation in which capacity withholding, e.g., by a large nuclear provider, creates an incentive for others to utilize their more expensive, yet flexible generation, such as gas plants.

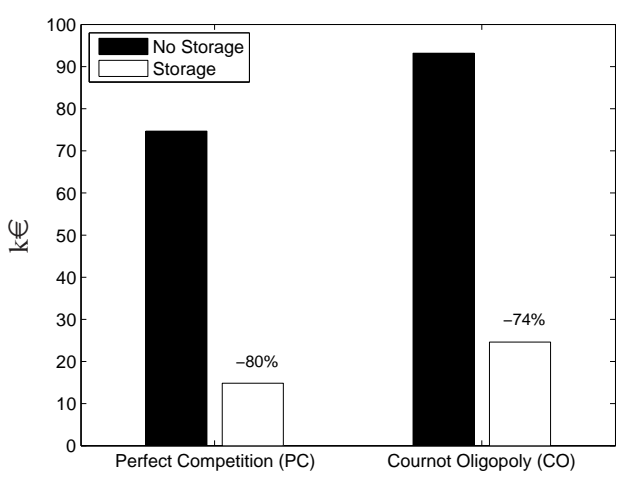

Fig. 7. Effect of storage on expected ramping costs (\%)

Since increased penetration of intermittent RE technologies can also pose problems for network congestion, we examine the expected congestion rent collected by the grid owner in Fig. 8. While storage alleviates network congestion, the effect is slightly stronger under PC, where total expected congestion charges decrease by $12 \%$. Under $\mathrm{CO}$, the expected congestion revenue is higher to begin with, and the alleviating effect is relatively smaller, i.e., 6\%. More network congestion under $\mathrm{CO}$ seems to result from strategically withheld sales (at nodes $n 2$ and $n 3$ in particular). This creates an incentive to increase imports and could cause congestion on relatively small crossborder lines such as the ones connecting nodes $n 3-n 5, n 6-$ $n 12$, and $n 11-n 15$ in Fig. 1.

Under PC, storage is used in a socially optimal way, i.e., for
TABLE III

TOTAL EXPECTED HOURLY POWER FLOWS (GW), INCLUDING THEIR SUM $(\Sigma)$, MEAN $(\bar{x})$, AND STANDARD DEVIATION $(\sigma)$ WITH DIFFERENCES BETWEEN NO-STORAGE AND STORAGE CASES INDICATED AS $\Delta$

\begin{tabular}{|l|c|c|c|c|c|c|}
\hline Hour & $\begin{array}{c}\text { PC } \\
\text { No storage }\end{array}$ & $\begin{array}{c}\text { PC } \\
\text { Storage }\end{array}$ & $\Delta$ & $\begin{array}{c}\text { CO } \\
\text { No Storage }\end{array}$ & $\begin{array}{c}\text { CO } \\
\text { Storage }\end{array}$ & $\Delta$ \\
\hline$t 5$ & 15.27 & 13.76 & -1.52 & 14.11 & 15.19 & 1.08 \\
$t 6$ & 14.41 & 13.43 & -0.98 & 14.84 & 14.80 & -0.04 \\
$t 7$ & 14.63 & 14.10 & -0.53 & 14.93 & 14.98 & 0.05 \\
$t 8$ & 14.13 & 14.74 & 0.61 & 14.67 & 14.41 & -0.26 \\
\hline$\Sigma$ & 58.45 & 56.03 & -2.41 & 58.55 & 59.38 & 0.82 \\
$\bar{x}$ & 14.61 & 14.01 & -0.60 & 14.64 & 14.84 & 0.21 \\
$\sigma$ & 0.49 & 0.56 & 0.07 & 0.37 & 0.33 & -0.04 \\
\hline
\end{tabular}

charging using the cheapest technology in off-peak hours in order to back up RE generation during peak hours. As a result, transmission flows decrease during off-peak hours because of the reduction in sales and are hardly affected during peak hours (Table III). Furthermore, flows under PC are generally from west to east, i.e., from $n 3$ to $n 5, n 12$ to $n 6$, and $n 15$ to $n 11$. Market power, however, reverses these flows because of the withholding of sales at $n 2, n 3$, and $n 6$ by EDF, which owns the most capacity. Under CO, EDF's withholding creates an opening for fringe firms from $n 1$, which are enticed by higher prices at $n 2, n 3$, and $n 6$. In fact, flows on the three aforementioned lines are reversed because of market power, and these flows actually increase because of the combined effect of market power and storage. A plausible explanation for the former outcome without storage is that EDF withholds nuclear production in $t 5$ only to ramp up at $t 6$ (Fig. 6b). This helps it to earn extraordinary profits from both its nuclear and hydro production even though ramping nuclear plants is costly. With storage, EDF produces up to its optimal level at $t 5$ without having to ramp up as much later on because it can divert "surplus" generation to storage. Hence, storage has a mild impact on congestion under $\mathrm{CO}$ partially because of the strategic use of storage by EDF, which entices German fringe producers to increase sales at nodes $n 2, n 3$, and $n 6$ during off-peak hours.

From Fig. 9, storage may increase expected $\mathrm{CO}_{2}$ emissions under $\mathrm{PC}$, but its effect is marginal under $\mathrm{CO}$. In spite 


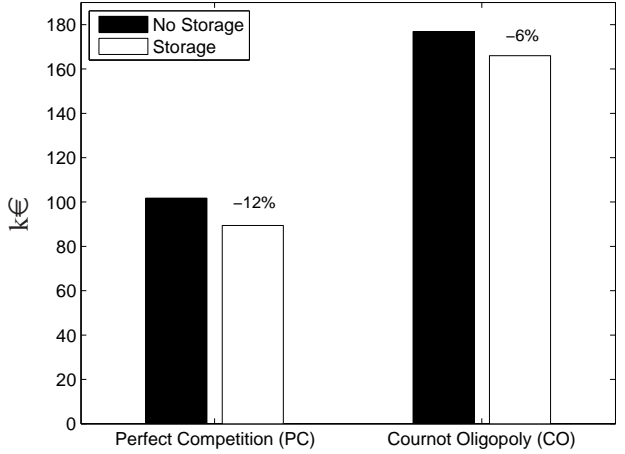

Fig. 8. Effect of storage on expected congestion revenues (\%)

of the emissions-increasing characteristic of storage operations, removing both storage and wind capacity from the grid would result in more fossil-fuel generation and, thus, increased emissions. As a benchmark, expected $\mathrm{CO}_{2}$ emissions would be $1.2 \%(\mathrm{PC})$ or $3.0 \%$ (CO) higher in a "no wind and storage" case than in the "wind and storage" case. The expected hourly $\mathrm{CO}_{2}$ emissions in Fig. 10 indicate that the undesirable effect of storage on $\mathrm{CO}_{2}$ emissions results from an increase in conventional generation during off-peak hours. When storage's efficiency losses are taken into account, the production decrease during peak hours is not enough to offset it, especially under PC. As seen in Fig. 6a, less polluting and more flexible gas production is substituted by cheaper but emission-increasing coal and by CCGT for off-peak storage charging. The difference between $\mathrm{PC}$ and $\mathrm{CO}$ can be attributed to Cournot producers' incentives to exploit their storage capacity to withhold sales. Finally, the results presented in this section are robust with respect to volatility in RE generation.

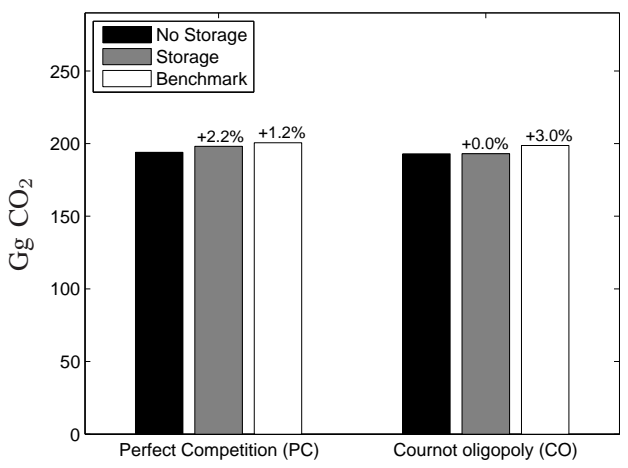

Fig. 9. Total expected $\mathrm{CO}_{2}$ emissions $(\mathrm{Gg})$

\section{Sensitivity Analysis with Respect to Nuclear Ramping}

We perform sensitivity analyses with respect to the nuclear ramp-up cost in order to examine its impact on the main results in the CO cases. Specifically, we increase the nuclear ramp-up cost in the $\mathrm{CO}(\mathrm{ns})$ and $\mathrm{CO}(\mathrm{s})$ cases until ramping by nuclear plants is eliminated. This occurs for ramp-up costs that are five times as high as the original, i.e., $€ 33.50 / \mathrm{MWh}$.

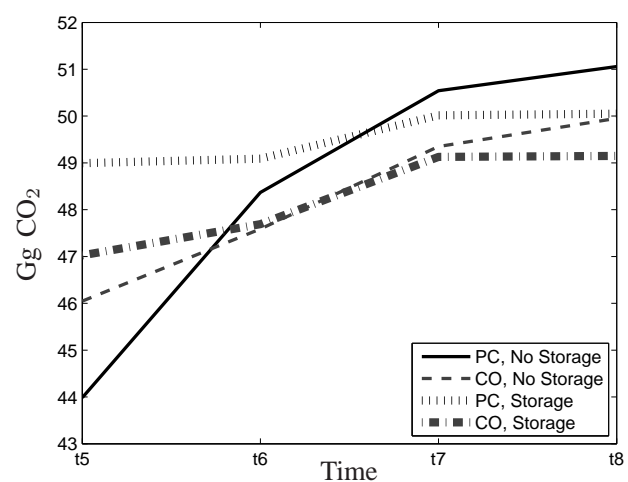

Fig. 10. Hourly expected $\mathrm{CO}_{2}$ emissions $(\mathrm{Gg})$

In spite of no ramping by nuclear plants in the $\mathrm{CO}$ cases, our results remain qualitatively unchanged. Furthermore, the counterintuitive impact of storage on expected emissions in the PC cases is not affected at all because there was no nuclear ramping to begin with in the absence of market power.

Specifically, after we eliminate nuclear ramping, the only aspect that changes is that nuclear generation is the same during off-peak and peak hours in both $\mathrm{CO}(\mathrm{ns})$ and $\mathrm{CO}(\mathrm{s})$ cases (Fig. 11). As with the original ramp-up costs, total expected nuclear generation is drastically reduced from approximately 260 GWh in the PC cases to 190 GWh in the CO cases, thereby resulting in similar consequences for expected prices, $\mathrm{CO}_{2}$ emissions, ramping costs, and congestion costs. While elimination of nuclear ramping is offset to some extent by increased gas and hydro ramping, expected ramping costs are lower to begin with in case $\mathrm{CO}(\mathrm{ns})$. Thus, the reduction in expected ramping costs as a result of storage under CO is $64 \%$ rather than $74 \%$ with the original ramp-up costs, which does not change the main finding in Fig. 7. Similarly, expected $\mathrm{CO}_{2}$ emissions were unaffected by storage in the $\mathrm{CO}$ cases using the original data and are now decreased by $0.1 \%$ with the elimination of nuclear ramping. Finally, prevalent transmission flows still go from east to west in the $\mathrm{CO}$ cases because of the withholding of nuclear generation relative to the PC cases. Table IV indicates that in spite of the elimination of nuclear ramping, expected flows still increase in off-peak hours due to storage because it enables EDF to exert market power to a greater extent. The reduction in expected congestion costs due to storage with the elimination of nuclear ramping under $\mathrm{CO}$ is $10 \%$ rather than $6 \%$ as with the original data (Fig. 8). This is because congestion is higher to begin with in the $\mathrm{CO}(\mathrm{ns})$ case as EDF reduces generation in peak hours, which creates more east-to-west transmission flows.

\section{Discussion AND CONCLUSIONS}

Electricity storage can contribute to increased energy sustainability by integrating increasing shares of intermittent $\mathrm{RE}$ in the power grid. Our examples show that in addition to previous findings on impacts of storage, e.g., its pricesmoothing effect and welfare benefits for society [9], [11], [13], it can alleviate network congestion and reduce producers' ramping of conventional generation. 

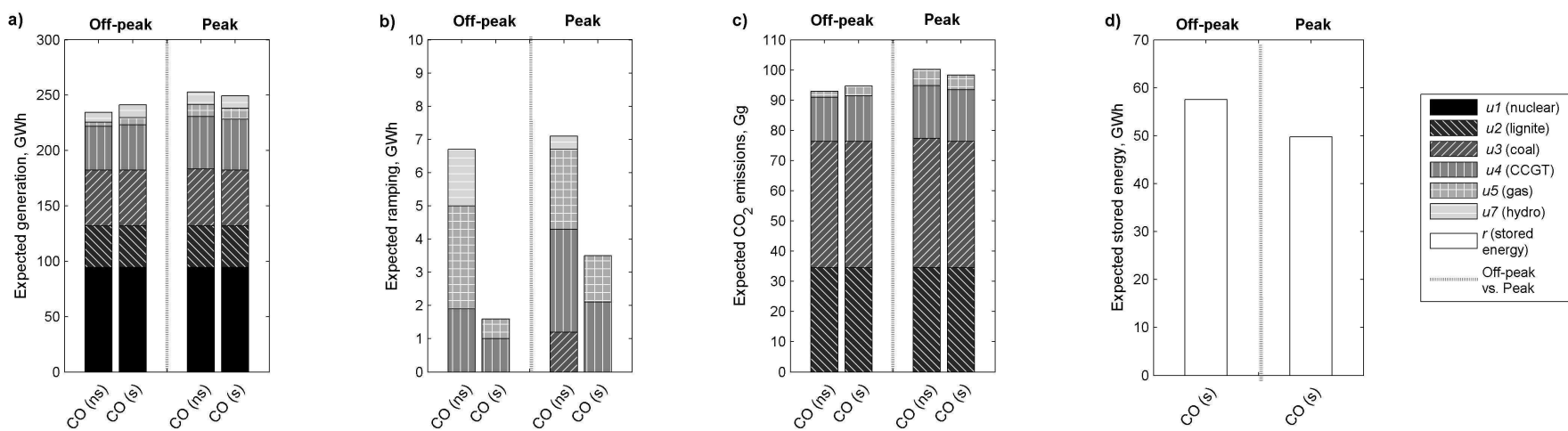

Fig. 11. Expected a) conventional generation (GWh), b) ramping (GWh), c) $\mathrm{CO}_{2}$ emissions $(\mathrm{Gg})$, and d) stored energy (GWh) in off-peak ( $\left.t 5-t 6\right)$ and peak hours $(t 7-t 8)$ for $\mathrm{CO}$ with (s) and without (ns) storage cases without nuclear ramping

TABLE IV

TOTAL EXPECTED HOURLY POWER FLOWS (GW), INCLUDING THEIR SUM

$(\Sigma)$, MEAN $(\bar{x})$, AND STANDARD DEVIATION $(\sigma)$ WITH DIFFERENCES BETWEEN NO-STORAGE AND STORAGE CASES INDICATED AS $\Delta$ WITHOUT NUCLEAR RAMPING

\begin{tabular}{|l|c|c|c|}
\hline Hour & $\begin{array}{c}\text { CO } \\
\text { No Storage }\end{array}$ & $\begin{array}{c}\text { CO } \\
\text { Storage }\end{array}$ & $\Delta$ \\
\hline$t 5$ & 14.12 & 15.18 & 1.07 \\
$t 6$ & 14.83 & 14.80 & -0.03 \\
$t 7$ & 15.02 & 15.00 & -0.02 \\
$t 8$ & 14.73 & 14.42 & -0.31 \\
\hline$\Sigma$ & 58.69 & 59.40 & 0.71 \\
$\bar{x}$ & 14.67 & 14.85 & 0.18 \\
$\sigma$ & 0.39 & 0.33 & -0.06 \\
\hline
\end{tabular}

We also demonstrate that storage may increase $\mathrm{CO}_{2}$ emissions especially in the case of perfect competition due to efficiency losses and changes in the generation mix. Interestingly, the effect of storage on $\mathrm{CO}_{2}$ emissions is negligible under market power as Cournot producers use storage strategically and not in a socially optimal manner. However, $\mathrm{CO}_{2}$ emissions are always lower than in a case with neither wind nor storage.

Some limitations of the modeling approach are the relatively short studied time frame due to the scenario tree analysis, the stylized and aggregated form of the network, and the absence of any restrictions on GHG emissions. Thus, extending our framework to include regulation to cap emissions by accounting for their social costs and investigating market design to find incentives for storage investments are important areas for future research. In the latter context, either a welfaremaximizing ISO or profit-maximizing strategic generators could invest in storage, thereby necessitating a bi-level programming approach [31], [32]. Finally, storage arbitrageurs in the Cournot oligopoly cases could be introduced since it is likely that standalone storage operation without generation would limit the extent to which strategic producers could exert market power [18].

\section{APPENDIX}

From (1)-(10), the producers' KKT conditions are:

$0 \leq-P_{s}\left[D_{t(s), n}^{\mathrm{int}}-D_{t(s), n}^{\mathrm{slp}}\left(\sum_{i^{\prime} \in \mathcal{I}} q_{s, n, i^{\prime}}+q_{s, n, i}\right)-\omega_{s, n}\right]$

$$
\begin{aligned}
& +\theta_{s i} \perp q_{s, n, i} \geq 0, \forall i, n, s \\
& 0 \leq P_{s}\left(C_{n, i, u}^{\text {conv }}-\omega_{s, n}\right)+\beta_{s, n, i, u}^{\text {conv }}+\beta_{s, n, i, u}^{\text {up }}-\sum_{s^{\prime} \in \mathcal{F}_{s}} \beta_{s^{\prime}, n, i, u}^{\text {up }} \\
& -\theta_{s, i} \perp g_{s, n, i, u}^{\mathrm{conv}} \geq 0, \forall i, n, s, \forall u \in \mathcal{U}_{n, i} \\
& 0 \leq P_{s} C_{n, i, u}^{\text {up }}-\beta_{s, n, i, u}^{\text {up }} \perp g_{s, n, i, u}^{\text {up }} \geq 0, \forall i, n, s, \forall u \in \mathcal{U}_{n, i} \\
& 0 \leq-P_{s} \omega_{s, n}-\theta_{s, i}+\beta_{s, n, i}^{e} \perp g_{s, n, i}^{e} \geq 0, \forall e, i, n, s \\
& 0 \leq P_{s} \omega_{s, n}+\theta_{s, i}-E^{\text {in }} \lambda_{s, n, i}^{\mathrm{bal}}+\lambda_{s, n, i}^{\text {in }} \perp r_{s, n, i}^{\text {in }} \geq 0, \forall i, n, s \\
& 0 \leq P_{s}\left(C^{\text {sto }}-\omega_{s, n}\right)-\theta_{s, i}+\lambda_{s, n, i}^{\text {bal }}+\lambda_{s, n, i}^{\text {out }} \\
& \perp r_{s, n, i}^{\text {out }} \geq 0, \forall i, n, s \\
& 0 \leq \lambda_{s, n, i}^{\mathrm{bal}}-\sum_{s^{\prime} \in \mathcal{F}_{s}} T_{t(s)} \cdot\left(1-E^{\mathrm{sto}}\right) \cdot \lambda_{s^{\prime}, n, i}^{\mathrm{bal}}+\lambda_{s, n, i}^{\mathrm{ub}}-\lambda_{s, n, i}^{\mathrm{lb}} \\
& \perp r_{s, n, i} \geq 0, \forall i, n, s \\
& \sum_{n \in \mathcal{N}} q_{s, n, i}-\sum_{n \in \mathcal{N}} \sum_{u \in \mathcal{U}_{n, i}} g_{s, n, i, u}^{\mathrm{conv}}-\sum_{n \in \mathcal{N}} \sum_{e \in \mathcal{E}} g_{s, n, i}^{e} \\
& -\sum_{n \in \mathcal{N}} r_{s, n, i}^{\text {out }}+\sum_{n \in \mathcal{N}} r_{s, n, i}^{\text {in }}=0 \text { with } \theta_{s, i} \text { u.r.s., } \forall i, s
\end{aligned}
$$$$
0 \leq T_{t(s)} \bar{G}_{n, i, u}^{\mathrm{conv}}-g_{s, n, i, u}^{\mathrm{conv}} \perp \beta_{s, n, i, u}^{\mathrm{conv}} \geq 0, \forall i, n, s, \forall u \in \mathcal{U}_{n, i}
$$$$
0 \leq-g_{s, n, i, u}^{\mathrm{conv}}+g_{a(s), n, i, u}^{\mathrm{conv}}+g_{s, n, i, u}^{\text {up }} \perp \beta_{s, n, i, u}^{\text {up }} \geq 0,
$$$$
\forall i, n, s, \forall u \in \mathcal{U}_{n, i}
$$$$
g_{s, n, i}^{e}-T_{t(s)} A_{s, n}^{e} \bar{G}_{n, i}^{e}=0 \text { with } \beta_{s, n, i}^{e} \text { u.r.s., } \forall e, i, n, s
$$$$
r_{s, n, i}-T_{t(s)}\left(1-E^{\text {sto }}\right) r_{a(s), n, i}-E^{\text {in }} r_{s, n, i}^{\text {in }}+r_{s, n, i}^{\text {out }}=0
$$$$
\text { with } \lambda_{s, n, i}^{\text {bal }} \text { u.r.s., } \forall i, n, s
$$$$
0 \leq T_{t(s)} \cdot R^{\text {in }} \cdot \bar{R}_{n, i}-r_{s, n, i}^{\text {in }} \perp \lambda_{s, n, i}^{\text {in }} \geq 0, \forall i, n, s
$$$$
0 \leq T_{t(s)} \cdot R^{\text {out }} \cdot \bar{R}_{n, i}-r_{s, n, i}^{\text {out }} \perp \lambda_{s, n, i}^{\text {out }} \geq 0, \forall i, n, s
$$$$
0 \leq \bar{R}_{n, i}-r_{s, n, i} \perp \lambda_{s, n, i}^{\mathrm{ub}} \geq 0, \forall i, n, s
$$$$
0 \leq-\underline{R}_{n, i}+r_{s, n, i} \perp \lambda_{s, n, i}^{\mathrm{lb}} \geq 0, \forall i, n, s .
$$

From (11)-(15), the grid owner's KKT conditions are:

$$
\begin{aligned}
& \sum_{n^{\prime} \in \mathcal{N}} P_{s} T_{t(s)} B_{n^{\prime}, n} \omega_{s, n^{\prime}}-\sum_{\ell \in \mathcal{L}} H_{\ell, n} \bar{\mu}_{s, \ell}+\sum_{\ell \in \mathcal{L}} H_{\ell, n} \underline{\mu}_{s, \ell} \\
& -S_{n} \gamma_{s, n}=0 \text { with } v_{s, n} \text { u.r.s., } \forall n, s
\end{aligned}
$$




$$
\begin{aligned}
& 0 \leq-\sum_{n \in \mathcal{N}} H_{\ell, n} v_{s, n}+K_{\ell} \perp \bar{\mu}_{s, \ell} \geq 0, \forall \ell, s \\
& 0 \leq \sum_{n \in \mathcal{N}} H_{\ell, n} v_{s, n}+K_{\ell} \perp \underline{\mu}_{s, \ell} \geq 0, \forall \ell, s \\
& S_{n} v_{s, n}=0 \text { with } \gamma_{s, n} \text { u.r.s., } \forall n, s .
\end{aligned}
$$

\section{ACKNOWLEDGMENT}

This paper has benefited from presentations at the $2015 \mathrm{BFG}$ Conference on Optimization and the 2015 European Conference on Operational Research as well as seminars at Aalto University and Berkeley Lab. Input from three anonymous referees has improved the paper. All remaining errors are the authors' own.

\section{REFERENCES}

[1] EC, "Directive 2009/28/EC of the European Parliament and of the Council of 23 April 2009 on the Promotion of the Use of Energy from Renewable Sources and Amending and Subsequently Repealing Directives 2001/77/EC and 2003/30/EC," Official Journal of the European Union, vol. L 140, pp. 16-62, 2009.

[2] — , "A Policy Framework for Climate and Energy in the Period from 2020 to 2030," European Commission, Communication from the Commission to the European Parliament, the Council, the European Economic and Social Committee and the Committee of the Regions $\operatorname{COM}(2014)$ 015, 2014.

[3] R. Wilson, "Architecture of Power Markets," Econometrica, vol. 70, no. 4, pp. 1299-1340, 2002.

[4] C. von Hirschhausen, "The German 'Energiewende' - An Introduction," Econ. Energy \& Environ. Policy, vol. 3, no. 2, pp. 1-12, 2014.

[5] L. Baringo and A. J. Conejo, "Transmission and Wind Power Investment," IEEE Trans. Power Syst., vol. 27, no. 2, pp. 885-893, 2012.

[6] L. Maurovich-Horvat, T. K. Boomsma, and A. S. Siddiqui, "Transmission and Wind Investment in a Deregulated Electricity Industry," IEEE Trans. Power Syst., vol. 30, no. 3, pp. 1633-1643, 2015.

[7] F. Kunz, "Improving Congestion Management: How to Facilitate the Integration of Renewable Generation in Germany," The Energy J., vol. 34 , no. 4, pp. 55-78, 2013

[8] A. Papavasiliou and S. S. Oren, "Large-Scale Integration of Deferrable Demand and Renewable Energy Sources," IEEE Trans. Power Syst., vol. 29, no. 1, pp. 489-499, 2014.

[9] W.-P. Schill and C. Kemfert, "Modeling Strategic Electricity Storage: The Case of Pumped Hydro Storage in Germany," The Energy J., vol. 32, no. 3, pp. 59-87, 2011.

[10] J. Bushnell, "A Mixed Complementarity Model of Hydrothermal Electricity Competition in the Western United States," Oper. Res., vol. 51, no. 1, pp. 80-93, 2003.

[11] R. Sioshansi, "When Energy Storage Reduces Social Welfare," Energy Econ., vol. 41, pp. 106-116, 2014.

[12] —, "Emissions Impacts of Wind and Energy Storage in a Market Environment," Environ. Sci. \& Tech., vol. 45, no. 24, pp. 10728-10735, 2011.

[13] A. S. A. Awad, J. D. Fuller, T. H. M. EL-Fouly, and M. M. A. Salama, "Impact of Energy Storage Systems on Electricity Market Equilibrium," IEEE Trans. Sust. Energy, vol. 5, no. 3, pp. 875-885, 2014.

[14] S. A. Gabriel, A. J. Conejo, J. D. Fuller, B. F. Hobbs, and C. Ruiz, Complementarity Modeling in Energy Markets. Springer, 2012.

[15] S. Wogrin and D. F. Gayme, "Optimizing Storage Siting, Sizing, and Technology Portfolios in Transmission-Constrained Networks," IEEE Trans. Power Syst., vol. PP, no. 99, pp. 1-10, 2015.

[16] R. Lueken and J. Apt, "The Effects of Bulk Electricity Storage on the PJM Market," Energy Syst., vol. 5, no. 4, pp. 677-704, 2014.

[17] Z. Hu and W. Jewell, "Optimal Power Flow Analysis of Energy Storage for Congestion Relief, Emissions Reduction, and Cost Savings." Phoenix, AZ, USA: IEEE PES Power Systems Conference and Exposition (PSCE), March 2011.

[18] B. F. Hobbs, "Linear Complementarity Models of Nash-Cournot Competition in Bilateral and POOLCO Power Markets," IEEE Trans. Power Syst., vol. 16, no. 2, pp. 194-202, 2001.

[19] S. A. Gabriel and F. U. Leuthold, "Solving Discretely-Constrained MPEC Problems with Applications in Electric Power Markets," Energy Econ., vol. 32, no. 1, pp. 3-14, 2010.
[20] A. Downward, "Carbon Charges in Electricity Markets with Strategic Behavior and Transmission," The Energy J., vol. 31, no. 4, pp. 159$166,2010$.

[21] European Energy Exchange, "EEX Actual Solar Generation," [Online]. Available: http://www.eex-transparency.com/homepage/ power/germany/production/usage/actual-solar-power-generation-/ actual-solar-power-generation-chart-

[22] - "EEX Actual Wind Generation." [Online]. Available: http://www.eex-transparency.com/homepage/power/germany/ production/usage/actual-wind-power-generation

[23] J. Egerer, C. Gerbaulet, R. Ihlenburg, F. Kunz, B. Reinhard, C. von Hirschhausen, A. Weber, and J. Weibezahn, "Electricity Sector Data for Policy-Relevant Modeling," Deutsches Institut für Wirtschaftsforschung, DIW Data Documentation 72, 2014

[24] F. U. Leuthold, "Economic Engineering Modeling of Liberalized Electricity Markets: Approaches, Algorithms, and Applications in a European Context," Ph.D. dissertation, Technische Universität Dresden, 2010.

[25] D. Huppmann and F. Kunz, "Introduction to Electricity Network Modelling." [Online]. Available: http://www.iot.ntnu.no/winterschool11/ web/material/ELMOD_Session_presentation.pdf

[26] D. Werner, "Electricity Market Price Volatility: The Importance of Ramping Costs," 2014, Working paper, Department of Agricultural and Resource Economics, University of Maryland, College Park.

[27] N. Kumar, P. Besuner, S. Lefton, D. Agan, and D. Hilleman, "Power Plant Cycling Costs," Intertek APTECH for the National Renewable Energy Laboratory (NREL) and Western Electricity Coordinating Council (WECC), Tech. Rep., 2012

[28] A. Lokhov, "Load-Following with Nuclear Power Plants," OECD Nuclear Energy Agency, NEA News 2011 No. 29.2, 2011. [Online]. Available: https://www.oecd-nea.org/nea-news/2011/29-2/ nea-news-29-2-load-following-e.pdf

[29] IPCC, "2006 IPCC Guidelines for National Greenhouse Gas Inventories," Intergovernmental Panel on Climate Change, International Guidelines, 2006.

[30] Sandia National Laboratories, "DOE Global Energy Storage Database." [Online]. Available: http://www.energystorageexchange.org/

[31] C. Ruiz, A. Conejo, and Y. Smeers, "Equilibria in an Oligopolistic Electricity Pool with Stepwise Offer Curves," IEEE Trans. Power Syst., vol. 27, no. 2, pp. 752-761, 2012.

[32] A. Zerrahn and D. Huppmann, "Network Expansion to Mitigate Market Power: How Increased Integration Fosters Welfare," DIW Berlin, Discussion Papers 1380, 2014.

Vilma Virasjoki received her M.Sc. degree in systems and operations research from Aalto University School of Science, Finland, in 2014. She is currently a doctoral student at the Systems Analysis Laboratory in the Department of Mathematics and Systems Analysis of Aalto University. Her research is on developing models for understanding and assessing how the increasing production of renewable energy impacts energy markets.

Paula Rocha completed the Ph.D. in Operations Research at Imperial College London in 2012 focusing on decision rules for deregulated electricity industries. Prior to her Ph.D. studies, she earned an M.Sc. in Statistics from University College London in 2007 and an undergraduate degree in Economics from the University of Porto. Her research is on developing tractable approximation schemes for optimizing large-scale energy models.

Afzal S. Siddiqui received the B.S. degree from Columbia University, New York, NY, USA, and the M.S. and Ph.D. degrees from the University of California, Berkeley, CA, USA, all in industrial engineering and operations research. He is a Senior Lecturer in the Department of Statistical Science at University College London and a Professor in the Department of Computer and Systems Sciences at Stockholm University. His research interests lie in distributed generation investment under uncertainty, real options analysis of technology adoption, and strategic interactions in capacity expansion.

Ahti Salo has worked extensively on decision analysis and its uses in resource allocation, innovation management, risk management, and efficiency analysis. He has published widely in leading international journals (including Management Science and Operations Research) and received awards for his research from the Decision Analysis Society of the Institute for Operations Research and the Management Sciences (INFORMS). He has been the President of the Finnish Operations Research Society for two biennial terms and is the Editor-in-Chief of the EURO Journal on Decision Processes. 